$8 \mathrm{RA}$ and $8 \mathrm{OA}$ patients undergoing hip replacement surgery, were utilised for gene-expression profiling by Affymetrix HG-U133Plus arrays. The BioRetis database was used for microarray analyses and generation of RA profiles.

Results Transcriptome analyses of synovial tissues from RA and OA patients revealed more than 1000 differentially expressed genes. Increased expression of genes involved in chemotaxis (CCL13, CCL18, CXCL9, CXCL10, CXCL13), cell adhesion and activation (ICAM1, PECAM1, ITGAL, ITGB2, CD40, CD86) indicate to inflammation but also to infiltration of various cell types like monocytes/macrophages, NK, T- and B-cells.

By comparing transcriptome of RA and OA monocytes, both from blood and bone marrow, it was obvious that monocytes were able to disclose differences between these two diseases. The RA disease-specific gene-expression profile was evident both in blood and bone marrow and it demonstrated only a minor overlap between these two bodies compartments. Altogether, a typical RA inflammatory profile disclosed in synovial tissues was greatly silenced in blood monocytes, and almost completely absent in bone marrow derived monocytes.

Conclusions The RA gene-expression profile was the most specific and robust in synovial tissue, demonstrating the dominance of the inflammatory process in the joints. Nevertheless, the systemic nature of RA was also evident at the level of blood and bone marrow monocytes. Concerning that blood is a favourable and easily accessible material for diagnosis and that monocytes are able to exhibit disease-specific alterations, understanding monocyte response in different rheumatic diseases seems to be advantageous approach for biomarkers discovery. This approach should be essential for identifying the objective criteria relevant for disease and therapeutic stratification of patients with RA.

\section{A10.7 DUAL EFFECTS OF SOLUBLE FASL AND MEMBRANE BOUND FASL ON FIBROBLAST-LIKE SYNOVIOCYTES CELLS (FLS) FROM RHEUMATOID ARTHRITIS (RA) PATIENTS}

doi:10.1136/annrheumdis-2013-203224.7

${ }^{2}$ Rachel Audo, ${ }^{2}$ Flavia Calmon-Hamaty, 'Bernard Combe, ${ }^{2}$ Michael Hahne, 1,2Jacques Morel. 'Department of Rheumatology, CHU Lapeyronie, Montpellier; ${ }^{2}$ IGMM, CNRS UMR5535, Montpellier

Background Membrane-bound FasL (mFasL) is able to induce fibroblast-like synoviocytes (FLS) apoptosis. In experimental arthritis mouse models, injection of agonistic antibody (Ab) anti-Fas decreased the symptoms. However, soluble FasL (sFasL) is increased in Rheumatoid Arthritis (RA) patients and correlated with disease activity. These results indicated that $\mathrm{mFasL}$ could be protective whereas sFasL could be deleterious suggesting that they could have different functions.

We analysed the effect of different FasL preparations mimicking sFasL or mFasL on RAFLS proliferation and apoptosis.

Methods RAFLS were treated with different FasL preparations (FasL-Flag $\pm \mathrm{Ab} \alpha$-Flag, FasL-Fc or sFasL) or with agonistic Ab antiFas. Apoptosis was then analysed by cytometry using annexinVFITC and TOPRO-3. Proliferation was measured using tritiated thymidine. Signaling pathways was analysed by western blot and their influence was assessed using chemical inhibitors. sFasL was quantified in synovial fluids from patients using cytometric bead array.

Results FasL-Flag alone (mimicking sFasL) was not able to induced FLS apoptosis while proliferation was significantly activated $(3.3 \pm$ 1 fold; $\mathrm{n}=5 ; \mathrm{p}<0.05)$. Similarly, sFasL was only able to strongly induce RAFLS proliferation $(7 \pm 5.5$ fold, $n=9 p<0.05)$. Membrane bound FasL (FasL-Flag + Ab $\alpha$-Flag) significantly induced RAFLS apoptosis $(52 \% \pm 18 ; n=5)$ and a slighter but significant proliferation $(2.2 \pm 0.3$ fold; $n=4)$. Duality of mFasL was confirmed using agonistic $\mathrm{Ab}$ anti-Fas (mimicking $\mathrm{mFasL}$ ) with pro-apoptotic $(38 \% \pm 18 ; n=2)$ and proliferative effect ( $4.4 \pm 2.0$ fold $)$. Finally, growing concentration of FasL-Fc leads to aggregation of the protein, mimicking mFas or sFasL at high and low concentration respectively. Dose responses confirmed $\mathrm{mFasL}$ and sFAsL effects. FasL activated Akt, JNK and ERK but also activated caspases $(n=5)$. Inhibition of each pathways block FasL-induced proliferation. However, only JNK inhibition significantly increased FasL-induced apoptosis. We observed that FasL-Fc was able to induce osteoarthritis (OA) FLS apoptosis but neither FasL-Fc nor sFAsL was able to significantly induced proliferation of OAFLS $(1.4 \pm 1.3$ and $2.6 \pm 1.1$ fold respectively, $n=4)$. Synovial fluids from patients with RA $(n=16)$ tends had higher sFasL concentrations compared to those with osteoarthritis $(n=10)(p=0.06)$.

Conclusions mFasL induces preferentially RAFLS apoptosis, whereas sFasL only induces RAFLS proliferation. Proliferative effect of sFasL was not seen on OAFLS. According to what we have already described for TRAIL, caspases are involved in FasL-induced apoptosis and proliferation. This is the first demonstration that sFasL and mFasL have different effects on RAFLS proliferation. sFasL by enhancing RAFLS proliferation could have a deleterious role in RA. Therefore, its blockage could be a therapeutic tool to prevent RA.

\section{A10.8 EVALUATION OF DISEASE ACTIVITY IN ADULT PATIENTS WITH JUVENILE IDIOPATHIC ARTHRITIS}

doi:10.1136/annrheumdis-2013-203224.8

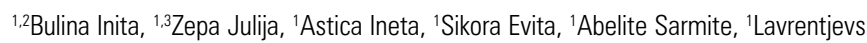
Vladimirs, ${ }^{1}{ }^{2}$ Andersone Daina. ${ }^{1}$ Pauls Stradins Clinical University Hospital; ${ }^{2}$ University of Latvia; ${ }^{3}$ Riga Stradins University, Riga, Latvia

Background Juvenile idiopathic arthritis (JIA) is a disease which maintains specific childish rheumatological features during whole life. There is still an open discussion which criteria of the disease activity should be used for the management of adult patients with JIA.

Objectives To analyse the usefulness of known disease activity and functional indices used in adult rheumatological practise for the assessment of rheumatoid arthritis (RA) and spondyloarthritidies (SpA): disease acitivity score (DAS), disease acitivity score 28 (DAS28), Bath Ankylosing Spondylitis Disease Activity Index (BASDAI), Bath Spondylitis Functional Index (BASFI), Health Assessment Questionnaire - disability index (HAQ-DI), short form health survey with 36 questions (SF-36).

Methods 35 adult patients with JIA (polyarticular form) classified by the ILAR (International League of Asociations for Rheumatology) classification criteria (Durban 1997, Edmonton 2001) were assessed clinically initially (I) and after 1 year (II) using DAS, DAS28, BASDAI, BASFI, HAQ-DI, SF-36.

Results 35 adult patients with JIA (polyarticular form) 19.4 (SD 1.8) years old with disease duration 6.5 (SD 4.2). 16 patients receive anti-TNF therapy and 19 patients methotrexate monotherapy. DAS 28 (I) 3.10 (IOR = 2.2) and DAS28 (II) 3.09 (IOR = 1.7), p=0.3; DAS (I) $2.09(\mathrm{IOR}=1.6)$ and DAS (II) $2.03(\mathrm{IOR}=1), \mathrm{p}=0.39$; HAO-DI (I) $0.44(\mathrm{IOR}=0.57)$ and HAQ-DI (II) $(\mathrm{IOR}=0.62), \mathrm{p}=0.32$; BASDAI (I) $3.6(\mathrm{IQR}=3.45)$ and BASDAI (II) 4.75 (IOR=3.275), $\mathrm{p}=0.46$; BASFI (I) 1.9 (3.15) and BASFI (II) 1.1 (IOR=1.2), $\mathrm{p}=0.057$; SF-36 physical health (I) $40.4(\mathrm{IOR}=9.5)$ and SF-36 physical health (II) 38.7 (IOR = 11), $p=0.02$; SF-36 mental health (I) $50.1(\mathrm{IOR}=27)$ and SF-36 mental health (II) $50.9(\mathrm{IOR}=14.2)$, $\mathrm{p}=0.6$.

Conclusions Accordingly to the results, increased values of disease activity indices (usually used in adults patients with RA and SpA) show that tendons as well as peripheral joints are involved in the inflammatory process of JIA polyarticular form. Therefore the evaluation of tendons/entheses in adult patients with JIA polyarticular 
form should be performed routinely in daily rheumatological practise including radiological assessment as well as standard evaluation of peripheral joints. Our data suggest that there is a necessity to create complex index which includes assessment of tendons/ entheses/peripheral joints for patients with JIA.

\section{A10.9 EVIDENCE FOR PROGRESSIVE REDUCTION AND LOSS OF TELOCYTES IN THE DERMAL CELLULAR NETWORK OF SYSTEMIC SCLEROSIS}

doi:10.1136/annrheumdis-2013-203224.9

1.2Mirko Manetti, ${ }^{2}$ Serena Guiducci, 'Martina Ruffo, 'Irene Rosa, 'Maria Simonetta Faussone-Pellegrini, 'Lidia Ibba-Manneschi, ${ }^{2}$ Marco Matucci-Cerinic. 'Department of Anatomy, Histology and Forensic Medicine, University of Florence, Florence, Italy; ${ }^{2}$ Department of Biomedicine, Division of Rheumatology, AOUC, and Excellence Centre for Research, Transfer and High Education DENO, The University of Florence, Florence, Italy

Background and Objectives Telocytes are a distinct population of stromal cells which have been recently identified in a wide variety of tissues and organs, including the skin. By their extremely long cytoplasmic processes telocytes may act as supporting cells and form a scaffold to define the correct three-dimensional organisation of tissues/organs during pre-natal life, or their repair/ renewal in post-natal life. Moreover, telocytes may influence the transcriptional activity of neighbouring stromal cells (fibroblasts/ myofibroblasts, mast cells), either by cell-to-cell contacts or by secreting paracrine signalling molecules, and may be implicated in tissue regeneration by cooperating with stem cell niches to form tandem cell structures. Systemic sclerosis (SSc) is a complex connective tissue disease characterised by fibrosis of the skin and internal organs. Up to now, most of the studies have focused on fibroblasts/myofibroblasts, while little is known about the possible involvement of other stromal cell types in SSc pathophysiology. In the present study, we investigated the distribution and ultrastructural features of telocytes in the skin of SSc patients compared with normal skin.

Methods Forearm skin biopsies were obtained from 24 SSc patients (13 limited cutaneous SSc (lcSSc), 11 diffuse cutaneous SSc (dcSSc)) and 10 healthy controls. Skin sections were subjected to immunoenzymatic or immunofluorescence labelling for CD34, CD31/ PECAM-1, alpha-smooth muscle actin (alpha-SMA), CD11c, CD90/ Thy-1, c-kit/CD117 and mast cell tryptase. Ultrathin sections were processed for transmission electron microscopy (TEM).

Results By an integrated immunohistochemical and TEM approach, we confirmed that telocytes were present in human dermis, where they were mainly recognisable by their typical ultrastructural features and were immunophenotypically characterised by CD34 expression. Dermal telocytes were immunophenotypically negative for CD31/PECAM-1 (endothelial cells), alpha-SMA (myofibroblasts, pericytes/vascular smooth muscle cells), CD11c (dendritic cells/macrophages), CD90/Thy-1 (fibroblasts) and c-kit/ CD117 (mast cells). In normal skin, telocytes were organised to form three-dimensional networks distributed among collagen bundles and elastic fibres, and surrounded microvessels, nerves and skin adnexa (hair follicles, sebaceous and sweat glands). Telocytes displayed severe ultrastructural damages (swollen mitochondria, cytoplasmic vacuolisation, lipofuscinic bodies) suggestive of ischaemia-induced cell degeneration and were progressively lost from the clinically affected skin of SSc patients. Telocyte damage and loss evolved differently according to $\mathrm{lcSSc} / \mathrm{dcSSc}$ subsets and early/advanced stages, being more rapid and severe in dcSSc.

Conclusions In SSc skin, the progressive loss of telocytes might i) contribute to the altered three-dimensional organisation of the extracellular matrix, ii) reduce the control of fibroblast/myofibroblast and mast cell activity, and iii) impair skin regeneration and/or repair.

\section{A10.10 EXAMINATION OF IL-6, TNF I AND II RECEPTOR DISTRIBUTION ON PERIPHERAL BLOOD MONONUCLEAR CELLS (PBMC) IN SEROPOSITIVE AND SERONEGATIVE RHEUMATOID ARTHRITIS (RA)}

doi:10.1136/annrheumdis-2013-203224.10

Martyna Skwarek, Babett Heschel, Julia Fantana, Martin Aringer. Division of Rheumatology, Department of Medicine III, University Clinical Center Carl Gustav Carus at the Technical University of Dresden, Germany

Background and Objectives Despite significant clinical overlap, genetic evidence and, in part, response to therapy distinguish seropositive $(R A+)$, i.e. rheumatoid factor $(R F)$ positive RA patients from patients with RF and anti-CCP-negative (RA-) disease. The aim of this study was to profile RA+ and RA- patients with regard to the differential expression of receptors for IL- 6 and TNF, cytokines of established importance in RA.

Materials and Methods PBMC of RA+ and RA- patients were compared to each other and to healthy individuals (HC). Most (93\%) of the RA+ patients were also positive for anti-CCP antibodies. PBMC were immediately prepared from peripheral venous blood. For determining the percentage of IL-6R $\alpha$ (CD126), gp130 (CD130), TNFR I (CD120a), and TNFR II (CD120b) positive cells, PBMC were stained with PE-labelled or control antibodies. Cells were analysed on a Becton Dickinson FACSCalibur fluorocytometer, gating for lymphocytes.

Results Disease duration (median 7 (0.04-66) versus 3.5 (0.04-6) years) and disease activity (CDAI median 15.6 (5.3-54.5) versus 13.5 (4.4-28)) were comparable between RA+ and RA- patients. Lymphocytes of RA+ and RA- patients differed in their lymphocyte expression of CD126+ and CD120b+. The percentage of CD126+ lymphocytes in RA+ was decreased in comparison with RA- (mean $\pm \mathrm{SD}, 49 \pm 14$ versus RA-58 $\pm 11, \mathrm{p}=0.05)$ and $\mathrm{HC}(59 \pm 9 \%$, $p=0.0007)$. The difference between RA- and HC was not significant. The percentage of CD130+ lymphocytes in RA+ (51 \pm 11$)$ was decreased when compared with $\mathrm{HC}(58 \pm 11 \%, \mathrm{p}=0.007)$. While the mean values for CD130 were similar between RA+ and RA- $(55 \pm 14 \%)$, the latter values were not significantly different from HC. In contrast to the IL-6 receptor, CD120b+ lymphocytes were increased in RA+ patients $(69 \pm 12 \%$ versus $R A-58 \pm 12 \%$, $p=0.04$, versus HC $57 \pm 11, p=0.0002)$. Again, the difference between RA- and HC was not significant. The percentages of CD120a + lymphocytes were low in all groups. Nevertheless, mean percentages of CD120a + lymphocytes from RA- (1.5 (0.53-2) \% versus HC (median $1(0.4-4) \% p=0.05)$ were somewhat higher than those of RA+ (median $1.1(0.3-3) \%, p=0.9)$.

Conclusions A direct comparison of (IL-6 mediated) downregulation of CD126 and (TNF mediated) upregulation of CD120b suggests that both are clearly more pronounced in RA+ than in RA-0. This was not explained by differences in disease activity.

\section{A10.11 EXPRESSION OF UNFOLDED PROTEIN RESPONSE GENES IN SYNOVIUM AND BLOOD MONONUCLEAR CELLS OF HLA-B27 POSITIVE ANKYLOSING SPONDYLITIS PATIENTS IS NOT INCREASED COMPARED TO OTHER ARTHRITIS PATIENTS AND HEALTHY CONTROLS}

doi:10.1136/annrheumdis-2013-203224.11

Barbara Neerinckx, Shea Carter, Rik J Lories. Laboratory of Tissue Homeostasis and Disease, Skeletal Biology and Engineering Research Center, Department of Development and Regeneration, KU Leuven, Belgium

Background The HLA-B27 heavy chain is prone to misfolding. Misfolded proteins give rise to endoplasmic reticulum stress and activation of the Unfolded Protein Response (UPR). The UPR is strongly activated in HLA-B27 transgenic rats, an animal model for 